\title{
Avaliação de estado nutricional da mangueira Tommy Atkins no submédio do vale do rio São Francisco: estabelecimento das normas DRIS
}

Recibido: 2 xuño 2008 / Aceptado: 12 setembro 2008

(C) IBADER- Universidade de Santiago de Compostela 2009

Resumo Este trabalho teve por objetivo estabelecer as normas do sistema integrado de diagnose e recomendação (DRIS) para manga, variedade Tommy Atkins. Foram avaliados sessenta e três pomares comerciais representativos do Submédio do Vale do Rio São Francisco, no Nordeste do Brasil. A região não dispõe de um conjunto de normas de diagnose de tecidos foliares para o cultivo, sendo o diagnóstico do estado nutricional $e$ as recomendações de fertilização baseados em faixas de teores de nutrientes em folhas de mangueira cultivadas em diferentes regiões. Foram coletadas amostras de folhas para análise química, antes da aplicação de quebradores de dormência de brotos. As normas DRIS para nutrição das plantas foram estabelecidas com base em um banco de dados criado nesta pesquisa, envolvendo os nutrientes $\mathrm{N}, \mathrm{P}$, $\mathrm{K}, \mathrm{Ca}, \mathrm{Mg}, \mathrm{B}, \mathrm{Cu}, \mathrm{Fe}, \mathrm{Mn}$ e $\mathrm{Zn}$. Essas normas foram

\section{P. A. da C. Pinto}

Prof. Pleno, Departamento de Tecnologia e Ciências Sociais, DTCS da Universidade do Estado da Bahia, UNEB, Cx. Postal 171, 48905680 Juazeiro - BA, Fone: (74) 36117363.

http://br.geocities.com/pacostapinto

e-mail: pacostapinto@hotmail.com

\section{E. Dias}

Prof. do Depto. de Solos da UFV, 36571000 Viçosa, MG. ledias@ufv.br

V. H. Alvarez V.

Prof. do Depto. de Solos da UFV, 36571000 Viçosa, MG. vhav@ufv.br

M. M. Choudhury

Pesquisador científico do CPATSA/EMBRAPA, Cx. Postal 23, 56 302970 Petrolina - PE. mohammad@cpatsa.embrapa.br

G. Vieira, Prof. do Depto. de Fitotecnia da UFV, 36570000 Viçosa, MG. gerival@ufv.br estabelecidas considerando uma subpopulação de plantas com produção igual ou superior a $250 \mathrm{~kg} /$ planta, produção essa representativa para as condições ambientais da principal zona produtora de manga do país. Das noventa relações consideradas entre nutrientes, sessenta e duas foram significativas pelo teste $F$, das quais, quarenta $e$ quatro foram selecionadas como normas para a cultura.

Palavras-chave Análise química de folhas · Nutrição de plantas - Diagnose

Summary: Evaluation of the nutritional estate of mango (Mangifera indica L.), variety Tommy Atkins, using DRIS in the Submédio of River São Francisco Valley: Norms Establishment. This work aimed to determine norms of the diagnosis and recommendation integrated system (DRIS) for mango crop, variety Tommy Atkins. Sixty three representative commercial orchards in the Submédio of River São Francisco Valley, in the Northeast of Brazil, were evaluated. The region does not make use of a set of norms of diagnose of leaves for the culture, being the diagnosis of the nutritional state and the recommendations of fertilization based in ranges of tenors of nutrients in leaves of mango in different regions. Samples of leaves were collected for chemical analysis, before the application of bud dormancy breaker. The DRIS norms for plants nutrition were established based on a database created in this research, with the nutrients $\mathrm{N}, \mathrm{P}, \mathrm{K}, \mathrm{Ca}, \mathrm{Mg}, \mathrm{B}, \mathrm{Cu}, \mathrm{Fe}, \mathrm{Mn}$ and $\mathrm{Zn}$. The norms, established from a population reference with production equal or superior to $250 \mathrm{~kg} / \mathrm{plant}$, are representative of the environmental conditions of the main producing zone of mango of the country, being applicable to the crop nutrition diagnosis. Of the ninety relationships considered among nutrients, sixty two were significant for the test $F$, and forty four of then have been selected as norms for the culture.

Key words: Chemical analyses leaves - Crop nutrition . Diagnosis 


\section{Introdução}

A fertilidade do solo é um dos mais importantes fatores determinantes da produção e da qualidade da manga (Raghupathi \& Bhargava, 1997). O uso correto de fertilizantes (quantidade, forma, época de aplicação e fonte), além de possibilitar menores custos na implantação e condução da cultura, minimiza a susceptibilidade das plantas a doenças e pragas, bem como o uso de agrotóxicos, reduzindo a possibilidade de poluição ambiental.

No entanto, resultados de análise de terra como única ferramenta para se fazer recomendação de fertilizantes apresentam limitada aplicabilidade para árvores frutíferas, devido à grande distribuição do sistema radicular, hábitos perenes, efeitos do porta-enxerto e frutificação diferenciada ao longo dos anos (Bhargava \& Chadha, 1988, citados por Samra \& Arora, 1997).

Com a grande extensão que o cultivo da mangueira ocupa na região sanfranciscana, há necessidade de se contar com um sistema eficiente de diagnóstico de desequilíbrios nutricionais dessa cultura, o que se reveste de grande importância econômica e ambiental.

De um modo geral as recomendações de fertilização da mangueira Tommy Atkins e de outras cultivares na região, têm sido baseadas em faixas de teores de nutrientes nas folhas de mangueiras cultivadas em outras regiões. Além disso, não se tem identificado a suficiência de cada nutriente em relação aos demais na planta.

Para tanto, é indispensável contar previamente com normas de diagnose do estado nutricional da cultura.

Devido às limitações dos conceitos de nível crítico ou das faixas de suficiência, o Sistema Integrado de Diagnose e Recomendação (DRIS) foi desenvolvido para preencher as expectativas de uso preditivo da diagnose foliar. Diferente de outros métodos, o DRIS é um procedimento integrado que identifica a suficiência de cada nutriente em relação aos outros na planta, ao invés de considerar apenas a concentração crítica de cada nutriente específico (Samra \& Arora, 1997).

O DRIS avalia o estado nutricional das plantas considerando o equilíbrio entre nutrientes, de modo que uma lavoura nutricionalmente equilibrada pode responder com alta produtividade, o que não acontece com lavouras com problemas de deficiência ou com desequilíbrios entre nutrientes (Sumner, 1999). O DRIS é um sistema de interpretação de resultados de análise de tecidos vegetais (Peverill, 1993; Reis Jr. (1999); Sumner, 1977; MeldalJohnsen \& Sumner, 1980; Jones Jr.,1993; Bailey et al.,1997), que foi desenvolvido para fornecer uma diagnose válida e classificar os nutrientes na sua ordem de limitação ao crescimento e desenvolvimento das plantas (Sumner, 1979).

Com o DRIS podem-se identificar alguns casos em que a produção está limitada por desequilíbrio nutricional, mesmo quando o teor de nenhum dos nutrientes esteja abaixo de seu nível crítico (Baldock \& Schulte, 1996); apresenta custo economicamente viável; permite a determinação simultânea de níveis de suficiência dos nutrientes para diferentes produtividades, nas melhores condições de balanço nutricional, a partir de dados obtidos em plantios comerciais (Sumner, 1999).

Algumas limitações do DRIS são: o sistema exige computação não muito simples (Baldock \& Schulte, 1996); não indica a probabilidade de resposta à adição do nutriente considerado limitante (Hallmark \& Beverly, 1991) e a dependência entre os índices, ou seja, o teor de um nutriente pode ter um efeito marcante sobre os outros índices (Baldock \& Schulte, 1996).

O DRIS baseia-se no cálculo de um índice para cada nutriente, comparando-se as relações entre um nutriente e cada um dos demais nutrientes na amostra sob diagnose com as relações envolvendo esse mesmo nutriente em uma população de alta produtividade. Conforme Alvarez V. \& Leite (1999) o índice DRIS de um determinado elemento $\left(I_{A}\right)$ é a média aritmética dos quocientes do teor deste nutriente $(A)$ com os teores dos demais nutrientes $(B, C, \ldots N)$ determinados na análise foliar. Estes quocientes (ou relações) ao mesmo tempo em que devem seguir distribuição normal, são reduzidos, ou seja, são transformados em varáveis normais reduzidas ( $\mathrm{z}$ ). Variáveis que são centradas e medidas com uma unidade igual ao seu desvio padrão (Vessereau, 1960, citado por Alvarez V. \& Leite 1999), portanto são quocientes estudentizados. Logo, $\mathrm{I}_{A}=\mathrm{z}=[\mathrm{z}$ $(A / B)+z(A / C)+\ldots+z(A / N)] /(n-1)$, onde $n$ é o número de nutrientes determinados na análise foliar. De acordo com Bailey et al. (1997) o índice DRIS de um nutriente nada mais é do que a média dos desvios das relações contendo um determinado nutriente em relação a seus respectivos valores ótimos. Cada relação entre teores de nutrientes nas lavouras de alta produtividade permite calcular as normas DRIS que são sua média, o desvio padrão e o coeficiente de variação. Índice DRIS negativo indica que o nutriente está abaixo do nível ótimo, enquanto que índice DRIS positivo indica que o nutriente está acima do nível ótimo (Baldock \& Schulte, 1996). Se o índice DRIS de um nutriente é igual a zero, este elemento é considerado como estando em perfeito equilíbrio com os outros nutrientes (Payne et al., 1990).

Schaffer et al. (1988) utilizaram o DRIS para identificar deficiências minerais associadas com declínio (desordem de etiologia desconhecida) de mangueiras Tommy Atkins, percebendo que o índice de desequilíbrio de nutrientes foi mais alto para árvores em pomares com altas percentagens de árvores em declínio do que naquelas em pomares geralmente saudáveis.

Raghupathi \& Bhargava (1999) realizaram trabalho em Ratnagiri, distrito de Maharashtra, Índia, para estudar o estado nutricional da manga Alphonso. As normas para classificação do 'status' de diferentes nutrientes em folhas foram desenvolvidas usando o DRIS. Reportaram que o teor foliar ótimo de $\mathrm{N}$ variou de 7,7 a 16,5, P de 0,224 a 0,336, $\mathrm{K}$ de 7,7 a 17,3, Ca de 7,6 a 16,3, S de 0,35 a 1,31 $\mathrm{g} \mathrm{kg}^{-1}$, Fe de 657 a 961, Mn de 13 a 408, Zn 7,71 a 18,3 e Cu de 14,3 a $17,8 \mathrm{mg} \mathrm{kg}^{-1}$. O nível de produção de 5,4 a $7,4 \mathrm{Mg}$ ha-1 foi possível na faixa ótima de nutrientes para essa variedade de manga desenvolvida nessa região. 
A concentração de nutrientes em folhas de manga, cv. Totapuri, afetada pela aplicação de níveis de nitrogênio, fósforo ou potássio foi monitorada em três experimentos, durante um período de nove anos, de 1991 a 1999. Os dados foram sub-divididos em população de baixa e de alta produtividade. Normas DRIS foram desenvolvidas a partir da população de alta produtividade, enquanto a diagnose de desequilíbrio de nutrientes foi feita com plantas de baixa produtividade. Quarenta e cinco expressões de nutrientes foram escolhidas como normas de diagnose. O maior desequilíbrio de nutrientes resultou em menor produção de frutos. Aplicou-se Análise dos Componentes Principais (PCA) para extrair a estrutura de correlação entre os nutrientes em plantas de baixa e de alta produtividade e dos índices DRIS (Raghupathi et al.,2004).

Hundal et al. (2005) utilizaram o DRIS para identificar o 'status' de nutrients em frutos de mangueiras em Punjab, India. Normas padrão foram estabelecidas a partir de frutos analisados, determinando índices de nutrientes nos frutos variando de 9,2-13,7, 0,8-1,6, 2,1-4,4, 17,1-34,7, 1,5-3,7 e 0,9-1,9 $\mathrm{g} \mathrm{kg}^{-1}$ para $\mathrm{N}, \mathrm{P}$, $\mathrm{K}, \mathrm{Ca}, \mathrm{Mg}$ e $\mathrm{S}$ e 11-19, 1-6, 63-227, 87-223 e 16-44 $\mathrm{mg} \mathrm{kg}^{-1}$ para $\mathrm{Zn}, \mathrm{Cu}, \mathrm{Fe}, \mathrm{Mn}$ e $\mathrm{B}$, respectivamente.

Raj \& Rao (2006) desenvolveram índices DRIS para identificar e priorizar os nutrients limitantes da produção em pomares de manga (cv. Baneshan) em Andhra Pradesh, India. As formas de expressão selecionadas para computação dos índices DRIS variaram desde árvores mais jovens $(<20$ anos de idade) até árvores com mais idade (> 20 anos). Os indices DRIS puderam refletir as variações a longo prazo de diferentes doses of nitrogenio, fósforo e potássio aplicados em manga, portanto demonstrando a sensibilidade dos recentes índices DRIS desenvolvidos para práticas de fertilização.

O objetivo deste trabalho foi estabelecer normas DRIS para a mangueira, cultivar Tommy Atkins, no submédio do vale do rio São Francisco, nordeste do Brasil.

\section{Material e métodos}

\section{Área de estudo}

O trabalho abrangeu os municípios de Juazeiro, Sobradinho, Casa Nova, Curaçá e Abaré, na Bahia e o de Petrolina, em Pernambuco, Pólo de maior destaque na exportação de mangas no Brasil.

O clima da região, segundo Köppen (Jacomine et al.,1976), é classificado como BSwh', quente, semi-árido, tipo estepe, com verão chuvoso, evapotranspiração elevada, sendo os meses de junho e de julho os de temperaturas mais amenas. A evapotranspiração potencial oscilou entre 1.800 e $2.000 \mathrm{~mm} / \mathrm{ano}$. No Quadro 1 são mostrados valores médios anuais de parâmteros agrometeorológicos da região nos anos de 1997 a 1999.
Amostragem e análise de material vegetal

A partir de um levantamento prévio, procurou-se selecionar propriedades agrícolas que representavam, em termos de manejo cultural e características de solos, o cultivo da mangueira Tommy Atkins na região.

No período de 1997 a 1999, em 63 propriedades agrícolas selecionadas, escolheu-se um talhão com árvores com sete ou mais anos de idade, porte uniforme e estado sanitário adequado. Amostras foliares foram obtidas ao longo de cada ano, na semana anterior à das pulverizações das copas com nitratos de potássio ou de cálcio, que são realizadas objetivando a quebra da dormência das gemas terminais. A amostragem de folhas antes dessa prática preveniu a contaminação da amostra com $\mathrm{N}, \mathrm{K}$ e, ou $\mathrm{Ca}$. As folhas foram coletadas na porção mediana da copa, nos quatro pontos cardeais (Quaggio, 1996), no penúltimo lançamento dos ramos, em vinte e cinco árvores do talhão. Foram selecionadas, no total, quatro folhas por árvore, perfazendo cem folhas por amostra composta, as quais foram acondicionadas em saco de papel e encaminhadas para análise em laboratório.

As folhas, depois de lavadas em água destilada, foram colocadas em estufa com circulação mecânica de ar, à temperatura variando de 65 a $70^{\circ} \mathrm{C}$ até peso constante, após o que foram moídas em moinho Wiley e passadas em peneira de $1 \mathrm{~mm}$ de malha.

$\mathrm{O} N$ foi determinado após digestão sulfúrica, pelo método micro Kjeldahl. Os elementos P, K, Ca, Mg, Cu, Fe, Mn e Zn foram determinados após digestão nítrico-perclórica. O $\mathrm{P}$ foi determinado por espectrofotometria com azul-de-molibdênio. $\mathrm{O}$ $\mathrm{Ca}$, o $\mathrm{Mg}$, o $\mathrm{Cu}$, o $\mathrm{Fe}$, o $\mathrm{Mn}$ e o $\mathrm{Zn}$ foram determinados empregando-se espectrofotômetro de absorção atômica. O K foi determinado por fotometria de chama (Malavolta et al.,1997). O B foi determinado, após incineração da amostra em forno elétrico a $550^{\circ} \mathrm{C}$, por colorimetria de curcumina (Malavolta et al.,1997).

Dada a relativa homogeneidade das árvores dos talhões, a produtividade foi estimada em t/ha em cada talhão pesquisado, sendo, em seguida, transformada em $\mathrm{kg} /$ planta, visto que na região há grande diversidade de populações de plantas por hectare, de 100 plantas a 278 plantas por hectare. Os dados de produtividade dos pomares amostrados foram registrados juntamente com os respectivos teores foliares de macro e micronutrientes, constituindo um banco de dados dividido em duas subamostras, uma de alta ( $\geq 250 \mathrm{~kg} /$ planta; 17 pomares) e outra de baixa (<250 kg/planta; 46 pomares) produtividade, representando duas subamostras. Os dados de teores foliares de nutrientes da mangueira foram processados pelo software Floresta 1.0 (Wadt, 1995).

As normas foram determinadas ao serem calculadas as médias, as variâncias $\left(\mathrm{S}^{2}\right)$ e os coeficientes de variação (CV) das relações quocientes entre os teores foliares de pares de nutrientes, testando-se a significância das mesmas pelo teste de $F$ para as variâncias, nas subamostras de alta e de baixa produtividade, maior sobre a menor ( $\mathrm{S}^{2}$ maior $/ \mathrm{S}^{2}$ menor), selecionando-se as de maior significância a até $10 \%$ de probabilidade. 


\begin{tabular}{|c|c|c|c|c|c|c|c|c|c|c|}
\hline Ano & Est. & $\begin{array}{l}\text { Precip. } \\
\text { (mm) }\end{array}$ & $\begin{array}{l}\text { Rad. } \\
\text { (ly/dia) }\end{array}$ & $\begin{array}{l}\text { Insol. } \\
\text { (h) }\end{array}$ & $\begin{array}{l}\text { Temp. } \\
\text { Média } \\
\left({ }^{\circ} \mathrm{C}\right)\end{array}$ & $\begin{array}{c}\text { Temp. } \\
\text { Mínima } \\
\left({ }^{\circ} \mathrm{C}\right)\end{array}$ & $\begin{array}{l}\text { Temp. } \\
\text { Máxima } \\
\left({ }^{\circ} \mathrm{C}\right)\end{array}$ & $\begin{array}{c}\text { Umidade } \\
\text { Relativa } \\
(\%)\end{array}$ & $\begin{array}{l}\text { Evap. } \\
\text { (mm/dia) }\end{array}$ & $\begin{array}{l}\text { Velocid. } \\
\text { Vento } \\
\text { (km/dia) }\end{array}$ \\
\hline \multirow{2}{*}{1997} & Mand. & 766,4 & 379,4 & 8,0 & 25,7 & 20,5 & 32,1 & 64 & 7,5 & 212,7 \\
\hline & Beb. & 629,2 & 433,4 & 7,4 & 25,7 & 21,3 & 32,2 & 68 & 6,7 & 152,9 \\
\hline \multirow{2}{*}{1998} & Mand. & 251,1 & 414,4 & 8,7 & 27,3 & 21,7 & 33,5 & 61 & 8,7 & 235,2 \\
\hline & Beb. & 394,9 & 456,5 & 8,1 & 27,3 & 23,6 & 33,6 & 74 & 7,8 & 195,4 \\
\hline \multirow{2}{*}{1999} & Mand. & 471,4 & 388,0 & 8,2 & 26,1 & 20,5 & 32,0 & 56 & 8,0 & 222,2 \\
\hline & Beb. & 493,6 & 416,7 & 7,4 & 25,9 & 21,2 & 32,3 & 69 & 7,1 & 187,5 \\
\hline
\end{tabular}

Fonte: EMBRAPA-CPATSA

Quadro 1.- Valores médios anuais de parâmteros agrometeorológicos coletados nas Estações de Mandacaru (Juazeiro - BA, 09²4'S, 40²6'W) e de Bebedouro (Petrolina - PE, 0909'S, 40²2'W) no período de 1997 a 1999.

\section{Resultados e discussão}

No Quadro 2 são apresentados os teores médios de macro e micronutrientes na matéria seca das folhas de mangueiras pertencentes aos pomares de alta e de baixa produtividade.

Quando se comparam as médias obtidas neste trabalho para cada nutriente contido na matéria seca foliar na subamostra de alta produtividade (Quadro 2), com as faixas de teores foliares utilizadas na região, constatam-se as seguintes porcentagens de equivalência entre a concentração obtida no presente trabalho em relação ao limite superior da faixa considerada adequada por Quaggio (1996): N (+6,86\%), P (- 26,88\%), K (- 17,5\%), Ca (- 28,8 $\%), \operatorname{Mg}(-46,2 \%), \mathrm{B}(+37,71 \%), \mathrm{Cu}(-46,84 \%)$, Fe $(-57,37$ $\%), \mathrm{Mn}(+547,46 \%)$ e Zn (- 46,1\%). Percebe-se, assim, que para $\mathrm{Fe}, \mathrm{Cu}, \mathrm{Mg}, \mathrm{Zn}, \mathrm{P}, \mathrm{Ca}$ e $\mathrm{K}$ as concentrações obtidas demonstram valores entre 17,5 e $57,37 \%$ inferiores ao valor máximo da faixa considerada adequada na região. Entretanto, as concentrações para N, B e Mn equivaleram a 6,86 a $547 \%$ superiores aos valores máximos considerados adequados na região. Estes três nutrientes têm sido aplicados com maior freqüência e em maior quantidade, seja nas adubações, como no caso de $\mathrm{N}$ e de $\mathrm{B}$, como em aplicações fitossanitárias incluindo $\mathrm{Mn}$. Deve-se considerar ainda o efeito da luz sobre a absorção deste último elemento, estimulando-a (Hewitt, 1963, citado por Camargo \& Silva, 1990), o que é perfeitamente possível na região, onde se constatam mais de 3.000 h/ano de luz solar.

Embora tratando de outras cultivares de manga plantada na Índia, os teores médios ótimos de nutrientes (em destaque no Quadro 2) encontrados neste trabalho em folhas de árvores de pomares de alta produtividade $(\geq 250 \mathrm{~kg} / \mathrm{planta}$, variando entre 25 e $50 \mathrm{Mg} \mathrm{ha}^{-1}$ ) - o que corresponde a cinco a sete vezes superiores às reportadas por Raghupathi \& Bhargava (1999) - se situaram dentro de faixas ou próximos aos seus limites superiores, conforme descrito por Raghupathi \& Bhargava (1999) e por Hundal et al. (2005) para o nitrogênio; por Hundal et al. (2005) para fósforo, cálcio, magnésio e ferro.

\begin{tabular}{|c|c|c|c|c|c|c|c|c|c|c|c|}
\hline Nutr. & $\begin{array}{c}\text { Sub- } \\
\text { Amostra }\end{array}$ & Média & $\mathrm{s}$ & $\mathrm{CV}$ & $\begin{array}{l}\left(\mathbf{S}^{2}{ }_{\text {maior }}\right. \\
\left.\mathbf{S}^{2}{ }_{\text {menor }}\right)\end{array}$ & Nutr. & $\begin{array}{c}\text { Sub- } \\
\text { Amostra }\end{array}$ & Média & s & CV & $\begin{array}{l}\left(\mathbf{S}^{2}{ }_{\text {maiorl }}\right. \\
\left.\mathbf{S}^{2}{ }_{\text {menor }}\right)\end{array}$ \\
\hline \multirow{3}{*}{$\mathrm{N}$} & & $\mathrm{g} \mathrm{kg}-1$ & & $\%$ & & & & $\mathrm{mg} \mathrm{kg}-1$ & & $\%$ & \\
\hline & A & 14,96 & 2,01 & 13,46 & \multirow{2}{*}{1,86} & \multirow{2}{*}{$\mathrm{Zn}$} & A & 21,56 & 15,40 & 71,45 & 1,02 \\
\hline & B & 14,71 & 2,74 & 18,62 & & & B & 22,22 & 15,21 & 68,45 & \\
\hline \multirow{2}{*}{$P$} & A & 1,17 & 0,26 & 21,87 & \multirow{2}{*}{3,70} & \multirow[b]{2}{*}{ B } & $A$ & 137,71 & 46,71 & 33,92 & 1,75 \\
\hline & B & 1,43 & 0,5 & 35,15 & & & B & 120,11 & 61,87 & 51,51 & \\
\hline \multirow{2}{*}{$\mathrm{K}$} & A & 8,25 & 2,18 & 26,36 & \multirow{2}{*}{1,57} & \multirow[t]{2}{*}{$\mathrm{Cu}$} & A & 26,58 & 20,26 & 76,25 & 18,49 \\
\hline & $B$ & 8,8 & 1,74 & 19,76 & & & $B$ & 57,83 & 87,11 & 150,65 & \\
\hline \multirow{2}{*}{$\mathrm{Ca}$} & A & 24,92 & 8,40 & 33,72 & \multirow{2}{*}{1,82} & \multirow[t]{2}{*}{$\mathrm{Fe}$} & A & 85,27 & 49,27 & 57,78 & 2,33 \\
\hline & B & 22,71 & 6,23 & 27,41 & & & $B$ & 125,36 & 75,26 & 60,04 & \\
\hline \multirow{2}{*}{$\mathrm{Mg}$} & A & 2,69 & 1,06 & 39,45 & \multirow{2}{*}{3,50} & \multirow{2}{*}{$\mathrm{Mn}$} & A & 647,46 & $\begin{array}{c}282,8 \\
0\end{array}$ & 43,68 & 1,36 \\
\hline & $B$ & 2,76 & 0,57 & 20,53 & & & B & 670,19 & $\begin{array}{c}329,6 \\
3\end{array}$ & 49,18 & \\
\hline
\end{tabular}

$A=$ Alta produtividade ( $\geq 250 \mathrm{~kg} /$ árvore $) ; B=$ Baixa produtividade ( $<250 \mathrm{~kg} /$ árvore $)$.
Quadro 2.- Teores médios de marco e micronutrientes na matéria seca das folhas, desvios padrão (S), coeficientes de variação (CV) e relação entre variâncias nas subamostras de alta (A) e de baixa (B) produtividade em pomares de mangueira Tommy Atkins 


\begin{tabular}{|c|c|c|c|c|c|c|c|c|c|}
\hline \multirow{2}{*}{ Relação } & \multicolumn{3}{|c|}{ Baixa } & \multicolumn{3}{|c|}{ Alta } & \multirow{2}{*}{$\left(\mathrm{S}_{\text {maior }}^{2} / \mathrm{S}_{\text {menor }}^{2}\right)$} & \multirow{2}{*}{ Significância } & \\
\hline & Média & $\mathbf{S}$ & $\mathrm{CV}$ & Média & $\mathbf{S}$ & $\mathrm{CV}$ & & & \\
\hline $\mathrm{N} / \mathrm{P}$ & 13,40 & 3,43 & 25,6 & 11,5 & 4,48 & 38,96 & 1,70 & 0 & \\
\hline $\mathrm{P} / \mathrm{N}$ & 0,08 & 0,02 & 24,14 & 0,10 & 0,04 & 38,5 & 4,00 & $\star * * *$ & \\
\hline $\mathrm{N} / \mathrm{K}$ & 1,96 & 0,7 & 35,61 & 1,72 & 0,42 & 24,6 & 2,78 & * & \\
\hline $\mathrm{K} / \mathrm{N}$ & 0,56 & 0,17 & 30,5 & 0,61 & 0,16 & 25,77 & 1,13 & NS & \\
\hline $\mathrm{N} / \mathrm{Ca}$ & 0,64 & 0,15 & 24,11 & 0,69 & 0,22 & 31,86 & 2,15 & * & \\
\hline $\mathrm{Ca} / \mathrm{N}$ & 1,66 & 0,47 & 28,23 & 1,59 & 0,56 & 34,97 & 1,42 & NS & \\
\hline $\mathrm{N} / \mathrm{Mg}$ & 6,37 & 3,07 & 48,17 & 5,54 & 1,47 & 26,58 & 4,36 & $* \star$ & \\
\hline $\mathrm{Mg} / \mathrm{N}$ & 0,18 & 0,07 & 38,5 & 0,19 & 0,06 & 28,74 & 1,36 & NS & \\
\hline N/B & 0,12 & 0,05 & 42,85 & 0,16 & 0,10 & 65,44 & 4,00 & $\star * * \star$ & \\
\hline $\mathrm{B} / \mathrm{N}$ & 9,25 & 2,93 & 31,71 & 8,49 & 4,90 & 57,64 & 2,80 & $* *$ & \\
\hline $\mathrm{N} / \mathrm{Zn}$ & 0,98 & 0,58 & 58,8 & 1,18 & 1,41 & 119,33 & 5,91 & 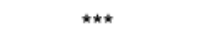 & \\
\hline $\mathrm{Zn} / \mathrm{N}$ & 1,42 & 0,94 & 66,19 & 1,55 & 1,08 & 69,53 & 1,32 & NS & \\
\hline $\mathrm{N} / \mathrm{Mn}$ & 0,03 & 0,03 & 91,13 & 0,03 & 0,02 & 67,53 & 2,25 & * & \\
\hline $\mathrm{Mn} / \mathrm{N}$ & 44,29 & 20,92 & 47,24 & 47,16 & 24,7 & 52,38 & 1,39 & NS & \\
\hline $\mathrm{N} / \mathrm{Fe}$ & 0,29 & 0,26 & 88,88 & 0,19 & 0,18 & 92,67 & 2,09 & 0 & \\
\hline Fé/N & 5,73 & 3,31 & 57,7 & 8,76 & 5,39 & 61,48 & 2,65 & $* *$ & \\
\hline $\mathrm{N} / \mathrm{Cu}$ & 1,14 & 1,1 & 96,09 & 0,95 & 1,31 & 137,62 & 1,42 & NS & \\
\hline $\mathrm{Cu} / \mathrm{N}$ & 1,79 & 1,29 & 72,31 & 3,88 & 5,54 & 142,6 & 18,44 & $\star \star * \star$ & \\
\hline $\mathrm{P} / \mathrm{K}$ & 0,15 & 0,04 & 24,55 & 0,17 & 0,06 & 36,98 & 2,25 & * & \\
\hline $\mathrm{K} / \mathrm{P}$ & 7,2 & 1,96 & 27,15 & 6,78 & 2,39 & 35,27 & 1,49 & NS & \\
\hline $\mathrm{P} / \mathrm{Ca}$ & 0,05 & 0,02 & 32,38 & 0,07 & 0,02 & 32,8 & 1,00 & NS & \\
\hline $\mathrm{Ca} / \mathrm{P}$ & 22,08 & 8,12 & 36,77 & 17,94 & 11,51 & 64,18 & 2,01 & * & \\
\hline $\mathrm{P} / \mathrm{Mg}$ & 0,48 & 0,18 & 37,03 & 0,53 & 0,20 & 38 & 1,23 & NS & \\
\hline $\mathrm{Mg} / \mathrm{P}$ & 2,38 & 0,96 & 40,13 & 2,12 & 0,73 & 34,51 & 1,73 & NS & \\
\hline $\mathrm{P} / \mathrm{B}$ & 0,01 & 0,01 & 56,17 & 0,02 & 0,01 & 76,44 & 1,00 & NS & \\
\hline $\mathrm{B} / \mathrm{P}$ & 121,85 & 39,59 & 32,49 & 95,24 & 60,33 & 63,35 & 2,32 & * & \\
\hline $\mathrm{P} / \mathrm{Zn}$ & 0,07 & 0,04 & 52,1 & 0,12 & 0,17 & 141,5 & 18,06 & 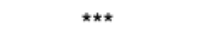 & \\
\hline $\mathrm{Zn} / \mathrm{P}$ & 17,87 & 10,42 & 58,34 & 17,1 & 13,3 & 77,78 & 1,63 & 0 & \\
\hline $\mathrm{P} / \mathrm{Mn}$ & 0,0012 & 0,0008 & 100,35 & 0,0026 & 0,0026 & 70,71 & 10,27 & $\star * * *$ & Quadro 3.- Média, \\
\hline $\mathrm{Mn} / \mathrm{P}$ & 587,74 & 304,25 & 51,77 & 551,25 & 407,8 & 73,98 & 1,80 & $\circ$ & $\begin{array}{l}\text { desvio padrāo }(\mathrm{S}) \text {, } \\
\text { coeficiente de }\end{array}$ \\
\hline $\mathrm{P} / \mathrm{Fe}$ & 0,02 & 0,02 & 74,43 & 0,02 & 0,02 & 88,03 & 1,00 & NS & $\begin{array}{l}\text { variação }(C V) \text { e } \\
\text { variância }(S 2) \text { das }\end{array}$ \\
\hline $\mathrm{Fe} / \mathrm{P}$ & 70,48 & 35,81 & 50,81 & 93,2 & 64,09 & 68,77 & 3,20 & $\star \star *$ & $\begin{array}{l}\text { relações entre teores } \\
\text { foliares de nutrientes }\end{array}$ \\
\hline $\mathrm{P} / \mathrm{Cu}$ & 0,09 & 0,10 & 114,75 & 0,09 & 0,11 & 117,27 & 1,21 & NS & obtidos para os teores \\
\hline $\mathrm{Cu} / \mathrm{P}$ & 22,18 & 15,18 & 68,44 & 50,66 & 86,59 & 170,93 & 32,54 & $\star * * *$ & $\begin{array}{l}\text { follares dos pares de } \\
\text { nutrientes da cultura }\end{array}$ \\
\hline $\mathrm{KJCa}$ & 0,36 & 0,12 & 34,02 & 0,41 & 0,13 & 32,40 & 1,17 & NS & $\begin{array}{l}\text { da mangueira Tommy } \\
\text { Atkins nas }\end{array}$ \\
\hline $\mathrm{Ca} / \mathrm{K}$ & 3,28 & 1,71 & 52,09 & 2,65 & 0,83 & 31,12 & 4,24 & ** & $\begin{array}{l}\text { subamostras de baixa } \\
\text { (B) e de alta (A) }\end{array}$ \\
\hline $\mathrm{K} / \mathrm{Mg}$ & 3,32 & 1,01 & 30,35 & 3,30 & 0,85 & 25,75 & 1,41 & NS & produtividade \\
\hline
\end{tabular}




\begin{tabular}{|c|c|c|c|c|c|c|c|c|c|}
\hline \multirow{2}{*}{ Relação } & \multicolumn{3}{|c|}{ Baixa } & \multicolumn{3}{|c|}{ Alta } & \multirow{2}{*}{$\left(\mathrm{S}_{\text {maior }}^{2} / \mathrm{S}_{\text {menor }}^{2}\right)$} & \multirow{2}{*}{ Significância } & \\
\hline & Média & $\mathbf{S}$ & CV & Média & $\mathbf{S}$ & $\mathrm{CV}$ & & & \\
\hline $\mathrm{Mg} / \mathrm{K}$ & 0,34 & 0,14 & 41,46 & 0,33 & 0,10 & 31,75 & 1,96 & 0 & \\
\hline K/B & 0,07 & 0,04 & 52,27 & 0,10 & 0,09 & 96,79 & 5,06 & $\star \star \star \star$ & \\
\hline $\mathrm{B} / \mathrm{K}$ & 17,82 & 7,28 & 40,89 & 14,06 & 7,11 & 50,56 & 1,05 & NS & \\
\hline $\mathrm{K} / \mathrm{Zn}$ & 0,53 & 0,36 & 67,32 & 0,68 & 0,69 & 102,39 & 3,67 & $\star \star \star \star$ & \\
\hline $\mathrm{Zn} / \mathrm{K}$ & 2,61 & 1,58 & 60,76 & 2,60 & 2,09 & 80,21 & 1,75 & $\circ$ & \\
\hline $\mathrm{K} / \mathrm{Mn}$ & 0,02 & 0,02 & 87,52 & 0,02 & 0,01 & 65,37 & 4,00 & ** & \\
\hline $\mathrm{Mn} / \mathrm{K}$ & 84,34 & 48,87 & 57,94 & 80,99 & 45,25 & 55,88 & 1,17 & NS & \\
\hline $\mathrm{K} / \mathrm{Fe}$ & 0,15 & 0,11 & 75,52 & 0,12 & 0,11 & 97,42 & 1,00 & NS & \\
\hline $\mathrm{Fe} / \mathrm{K}$ & 10,35 & 6,19 & 59,78 & 15,03 & 9,71 & 64,58 & 2,46 & $\star \star \star$ & \\
\hline $\mathrm{K} / \mathrm{Cu}$ & 0,61 & 0,58 & 94,36 & 0,56 & 0,64 & 113,1 & 1,22 & NS & \\
\hline $\mathrm{Cu} / \mathrm{K}$ & 3,36 & 2,50 & 74,63 & 7,21 & 11,25 & 155,98 & 20,25 & 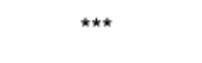 & \\
\hline $\mathrm{Ca} / \mathrm{Mg}$ & 10,56 & 6,80 & 64,38 & 8,67 & 3,85 & 44,46 & 3,12 & $\star \star$ & \\
\hline $\mathrm{Mg} / \mathrm{Ca}$ & 0,11 & 0,04 & 35,81 & 0,13 & 0,05 & 35,64 & 1,56 & NS & \\
\hline $\mathrm{Ca} / \mathrm{B}$ & 0,19 & 0,06 & 33,65 & 0,23 & 0,12 & 51,38 & 4,00 & $\star \star \star \star ~$ & \\
\hline $\mathrm{B} / \mathrm{Ca}$ & 5,73 & 1,78 & 31,16 & 5,39 & 2,55 & 47,30 & 2,05 & $\circ$ & \\
\hline $\mathrm{Ca} / \mathrm{Zn}$ & 1,65 & 1,03 & 62,52 & 1,88 & 2,29 & 121,71 & 4,94 & $\star \star \star \star$ & \\
\hline $\mathrm{Zn} / \mathrm{Ca}$ & 0,96 & 0,81 & 84,94 & 1,08 & 0,84 & 77,58 & 1,07 & NS & \\
\hline $\mathrm{Ca} / \mathrm{Mn}$ & 0,06 & 0,09 & 144,28 & 0,04 & 0,03 & 66,60 & 9,00 & $\star \star \star \star$ & \\
\hline $\mathrm{Mn} / \mathrm{Ca}$ & 29,51 & 16,53 & 56,03 & 31,33 & 16,27 & 51,92 & 1,03 & NS & \\
\hline $\mathrm{Ca} / \mathrm{Fe}$ & 0,49 & 0,46 & 93,49 & 0,28 & 0,23 & 82,64 & 4,00 & $\star \star$ & \\
\hline Fé/Ca & 3,69 & 2,31 & 62,63 & 5,83 & 3,82 & 65,48 & 2,73 & $\star *$ & \\
\hline $\mathrm{Ca} / \mathrm{Cu}$ & 1,89 & 1,88 & 99,3 & 1,45 & 1,79 & 123,38 & 1,10 & NS & \\
\hline $\mathrm{Cu} / \mathrm{Ca}$ & 1,17 & 0,95 & 81,19 & 2,65 & 3,89 & 146,84 & 16,77 & $\star \star * \star$ & \\
\hline $\mathrm{Mg} / \mathrm{B}$ & 0,02 & 0,01 & 44,59 & 0,03 & 0,02 & 70,81 & 4,00 & 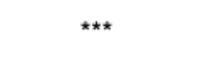 & \\
\hline $\mathrm{B} / \mathrm{Mg}$ & 54,69 & 19,71 & 36,05 & 45,21 & 24,73 & 54,70 & 1,57 & NS & \\
\hline $\mathrm{Mg} / \mathrm{Zn}$ & 0,19 & 0,15 & 80,6 & 0,23 & 0,31 & 133,56 & 4,27 & $\star \star * *$ & \\
\hline $\mathrm{Zn} / \mathrm{Mg}$ & 9,06 & 7,06 & 77,98 & 8,32 & 5,54 & 66,54 & 1,62 & NS & \\
\hline $\mathrm{Mg} / \mathrm{Mn}$ & 0,01 & 0,01 & 166,41 & 0,01 & 0,00 & 64,95 & 0,00 & NS & \\
\hline $\mathrm{Mn} / \mathrm{Mg}$ & 263,57 & 106,95 & 40,58 & 252,68 & 140,58 & 55,64 & 1,73 & ○ & \\
\hline $\mathrm{Mg} / \mathrm{Fe}$ & 0,05 & 0,05 & 96,24 & 0,04 & 0,03 & 89,36 & 2,78 & * & $\begin{array}{l}\text { Quadro } 3 \text { cont.- } \\
\text { Média, desvio padrão }\end{array}$ \\
\hline $\mathrm{Fe} / \mathrm{Mg}$ & 34,65 & 22,49 & 64,92 & 46,83 & 30,29 & 64,68 & 1,81 & $\circ$ & $\begin{array}{l}\text { (S), coeficiente de } \\
\text { variação }(C V) \text { e }\end{array}$ \\
\hline $\mathrm{Mg} / \mathrm{Cu}$ & 0,20 & 0,19 & 96,38 & 0,18 & 0,21 & 115,84 & 1,22 & NS & $\begin{array}{l}\text { variância (S2) das } \\
\text { relações entre teores }\end{array}$ \\
\hline $\mathrm{Cu} / \mathrm{Mg}$ & 11,19 & 9,87 & 88,28 & 23,66 & 39,26 & 165,94 & 15,82 & $\star \star \star \star$ & foliares de nutrientes \\
\hline $\mathrm{B} / \mathrm{Zn}$ & 9,30 & 6,01 & 64,68 & 9,98 & 12,43 & 124,62 & 4,28 & $\star \star \star *$ & $\begin{array}{l}\text { obtidos para os teores } \\
\text { foliares dos pares de }\end{array}$ \\
\hline $\mathrm{Zn} / \mathrm{B}$ & 0,19 & 0,18 & 97,35 & 0,26 & 0,32 & 123,78 & 3,16 & $\star \star *$ & $\begin{array}{l}\text { nutrientes da cultura } \\
\text { da mangueira Tommy }\end{array}$ \\
\hline $\mathrm{B} / \mathrm{Mn}$ & 0,32 & 0,45 & 139,54 & 0,25 & 0,25 & 101,34 & 3,24 & $\star \star *$ & $\begin{array}{l}\text { Atkins nas } \\
\text { subamostras de baixa }\end{array}$ \\
\hline $\mathrm{Mn} / \mathrm{B}$ & 5,09 & 2,15 & 42,18 & 6,79 & 4,09 & 60,27 & 3,62 & $* \star \star *$ & $\begin{array}{l}\text { (B) e de alta (A) } \\
\text { produtividade }\end{array}$ \\
\hline $\mathrm{B} / \mathrm{Fe}$ & 2,67 & 2,29 & 85,9 & 1,64 & 1,64 & 99,59 & 1,95 & 0 & \\
\hline
\end{tabular}




\begin{tabular}{|c|c|c|c|c|c|c|c|c|}
\hline \multirow{2}{*}{ Relação } & \multicolumn{3}{|c|}{ Baixa } & \multicolumn{3}{|c|}{ Alta } & \multirow{2}{*}{$\left(\mathrm{S}_{\text {maior }}^{2} / \mathrm{S}_{\text {menor }}^{2}\right)$} & \multirow{2}{*}{ Significância } \\
\hline & Média & $\mathbf{S}$ & $\mathrm{CV}$ & Média & $\mathbf{S}$ & $\mathrm{CV}$ & & \\
\hline $\mathrm{Fe} / \mathrm{B}$ & 0,73 & 0,62 & 85,43 & 1,47 & 1,62 & 109,68 & 6,83 & $\star \star \star \star$ \\
\hline $\mathrm{B} / \mathrm{Cu}$ & 10,52 & 10,36 & 98,46 & 9,77 & 22,49 & 230,14 & 4,71 & $\star \star \star \star$ \\
\hline $\mathrm{Cu} / \mathrm{B}$ & 0,23 & 0,22 & 94,56 & 0,56 & 0,79 & 141,54 & 12,89 & $\star \star \star$ \\
\hline $\mathrm{Zn} / \mathrm{Fe}$ & 0,31 & 0,19 & 61,78 & 0,27 & 0,27 & 101,57 & 2,02 & ० \\
\hline $\mathrm{Fe} / \mathrm{Zn}$ & 4,61 & 2,78 & 60,38 & 9,92 & 15,22 & 153,4 & 29,97 & $\star \star \star \star$ \\
\hline $\mathrm{Zn} / \mathrm{Cu}$ & 1,45 & 1,82 & 125,29 & 1,32 & 1,80 & 136,1 & 1,02 & NS \\
\hline $\mathrm{Cu} / \mathrm{Zn}$ & 1,49 & 1,12 & 74,70 & 4,19 & 7,70 & 183,9 & 47,26 & $\star \star \star *$ \\
\hline $\mathrm{Mn} / \mathrm{Zn}$ & 43,77 & 38,06 & 86,95 & 60,43 & 102,63 & 169,84 & 7,27 & $\star \star \star \star ~$ \\
\hline $\mathrm{Zn} / \mathrm{Mn}$ & 0,04 & 0,03 & 73,70 & 0,05 & 0,04 & 85,25 & 1,78 & ० \\
\hline $\mathrm{Mn} / \mathrm{Fe}$ & 13,82 & 17,09 & 123,69 & 8,34 & 8,50 & 101,91 & 4,04 & $\star \star$ \\
\hline $\mathrm{Fe} / \mathrm{Mn}$ & 0,19 & 0,23 & 120,85 & 0,23 & 0,16 & 70,54 & 2,07 & o \\
\hline $\mathrm{Mn} / \mathrm{Cu}$ & 56,86 & 69,95 & 123,02 & 43,34 & 48,52 & 111,97 & 2,08 & ० \\
\hline $\mathrm{Cu} / \mathrm{Mn}$ & 0,07 & 0,08 & 130,14 & 0,12 & 0,22 & 185,65 & 7,56 & $\star \star \star$ \\
\hline $\mathrm{Fe} / \mathrm{Cu}$ & 6,62 & 13,05 & 197,13 & 7,92 & 9,41 & 118,88 & 1,92 & 0 \\
\hline $\mathrm{Cu} / \mathrm{Fe}$ & 0,36 & 0,21 & 58,76 & 0,73 & 1,23 & 169,74 & 34,31 & $\star \star \star$ \\
\hline
\end{tabular}

Variâncias das subamostras de alta e baixa produtividade estatisticamente diferentes a $0,1\left(^{\star \star \star}\right), 1,0\left({ }^{\star \star}\right), 5,0$

(*) ou $10 \%$ (o) de probabilidade; $N$ = Não Significativo, a 10 \% (teste F). A razão entre variâncias foi

estabelecida a partir da divisão da maior sobre a menor.
Quadro 3 cont.-

Média, desvio padrão (S), coeficiente de variação $(C V)$ e variância (S2) das relações entre teores foliares de nutrientes obtidos para os teores foliares dos pares de nutrientes da cultura da mangueira Tommy Atkins nas subamostras de baixa (B) e de alta (A) produtividade
Os coeficientes de variação para os teores de nutrientes foliares foram maiores na subamostra de baixa produtividade para a maioria $(60 \%)$ dos elementos analisados: N, P, B, Cu, Fe e Mn (Quadro 2). Tal fato deve estar associado à diversidade de solos nos diferentes pomares, sistemas de manejo, incluindo espaçamentos, fertilizações, controles fitossanitários e quantidade de água de irrigação.

Conforme Walworth \& Sumner (1987), o coeficiente de variação $(\mathrm{CV})$ das variáveis obtidas das análises de uma população de plantas reflete a variação real dos respectivos valores. Os desvios padrões permitem determinar a faixa acima e abaixo da norma na qual se considera que o nutriente em pauta se encontra em um balanço de adequada concentração e, portanto, de adequado estado nutricional. Os valores de $\mathrm{CV}$ das relações de teores foliares nos pomares de baixa produtividade variaram de $24,11 \%(\mathrm{~N} / \mathrm{Ca})$ a $197,13 \%$ (Fe/Cu) (Quadro 3).

As relações $\mathrm{Cu} / \mathrm{N}, \mathrm{Cu} / \mathrm{P}, \mathrm{Cu} / \mathrm{K}, \mathrm{Cu} / \mathrm{Ca}, \mathrm{Cu} / \mathrm{Mg}, \mathrm{Cu} / \mathrm{B}$, $\mathrm{Cu} / \mathrm{Zn}, \mathrm{Cu} / \mathrm{Fe}, \mathrm{Fe} / \mathrm{Zn}$ e $\mathrm{P} / \mathrm{Zn}$ (Quadro 3) apresentaram valores de $\mathrm{CV}$ consistentemente mais altos nos pomares de baixa produtividade, resultando em relações entre variâncias ( $\mathrm{S}^{2}{ }_{\text {maior }} / \mathrm{S}^{2}$ menor $)$ altas, entre 12,9 e 47,3, diferençando significativamente as duas subamostras. $O$
Cobre é absorvido como $\mathrm{Cu}^{2+}$ e $\mathrm{Cu}$-quelato e a concentração do mesmo nas plantas é pequena, geralmente entre 2 e $20 \mathrm{mg} \mathrm{kg}^{-1}$ de matéria seca. A sua absorção parece ocorrer por processo ativo e existem evidências de que este elemento inibe fortemente a absorção do $\mathrm{Zn}$ e vice-versa (Bowen, 1969, citado por Dechen et al., 1991). Os teores foliares de $\mathrm{Cu}$, nos pomares de baixa e de alta produtividade, variaram de 2,0 a 381,0 e de 3,4 a $70,0 \mathrm{mg} \mathrm{kg}^{-1}$ de matéria seca, enquanto que o CV alcançou os valores máximos de 150,6 e 76,25, respectivamente, sendo esses os mais altos valores de CV encontrados entre os nutrientes considerados. A menor amplitude de variação dos teores de $\mathrm{Cu}$ nos pomares de alta produtividade deve decorrer de um manejo mais criterioso desse e de outros nutrientes. Acredita-se que, nos pomares de baixa $(19,56 \%)$ e de alta $(23,52 \%)$ produtividade, a utilização de $\mathrm{Cu}$ em controles fitossanitários empregando produtos à base desse nutriente, tais como oxicloreto de cobre e óxido cuproso, seja responsável pelos excessos de cobre constatados em folhas.

O cobre tem forte afinidade com o átomo de nitrogênio do grupo amino, acreditando-se que compostos nitrogenados solúveis, como os aminoácidos, atuam como carregadores desse nutriente no xilema e no floema (Lonegragan, 1981, citado por Dechen et al. 1991). 


\begin{tabular}{cccccccc}
\hline Relaçao & Média & $\mathbf{S}$ & $\mathrm{CV}$ & Relaçao & Média & $\mathbf{S}$ & CV \\
\hline $\mathrm{P} / \mathrm{N}$ & 0,10 & 0,04 & 38,5 & $\mathrm{Cu} / \mathrm{K}$ & 7,21 & 11,25 & 155,98 \\
$\mathrm{~N} / \mathrm{K}$ & 1,72 & 0,42 & 24,6 & $\mathrm{Ca} / \mathrm{Mg}$ & 8,67 & 3,85 & 44,46 \\
$\mathrm{~N} / \mathrm{Ca}$ & 0,69 & 0,22 & 31,86 & $\mathrm{Ca} / \mathrm{B}$ & 0,23 & 0,12 & 51,38 \\
$\mathrm{~N} / \mathrm{Mg}$ & 5,54 & 1,47 & 26,58 & $\mathrm{Ca} / \mathrm{Zn}$ & 1,88 & 2,29 & 121,71 \\
$\mathrm{~N} / \mathrm{B}$ & 0,16 & 0,10 & 65,44 & $\mathrm{Ca} / \mathrm{Mn}$ & 0,04 & 0,03 & 66,60 \\
$\mathrm{~N} / \mathrm{Zn}$ & 1,18 & 1,41 & 119,33 & $\mathrm{Ca} / \mathrm{Fe}$ & 0,28 & 0,23 & 82,64 \\
$\mathrm{~N} / \mathrm{Mn}$ & 0,03 & 0,02 & 67,53 & $\mathrm{Cu} / \mathrm{Ca}$ & 2,65 & 3,89 & 146,84 \\
$\mathrm{Fé} / \mathrm{N}$ & 8,76 & 5,39 & 61,48 & $\mathrm{Mg} / \mathrm{B}$ & 0,03 & 0,02 & 70,81 \\
$\mathrm{Cu} / \mathrm{N}$ & 3,88 & 5,54 & 142,6 & $\mathrm{Mg} / \mathrm{Zn}$ & 0,23 & 0,31 & 133,56 \\
$\mathrm{P} / \mathrm{K}$ & 0,17 & 0,06 & 36,98 & $\mathrm{Mn} / \mathrm{Mg}$ & 252,68 & 140,58 & 55,64 \\
$\mathrm{Ca} / \mathrm{P}$ & 17,94 & 11,51 & 64,18 & $\mathrm{Fe} / \mathrm{Mg}$ & 46,83 & 30,29 & 64,68 \\
$\mathrm{~B} / \mathrm{P}$ & 95,24 & 60,33 & 63,35 & $\mathrm{Cu} / \mathrm{Mg}$ & 23,66 & 39,26 & 165,94 \\
$\mathrm{P} / \mathrm{Zn}$ & 0,12 & 0,17 & 141,5 & $\mathrm{~B} / \mathrm{Zn}$ & 9,98 & 12,43 & 124,62 \\
$\mathrm{P} / \mathrm{Mn}$ & 0,0026 & 0,0026 & 70,71 & $\mathrm{Mn} / \mathrm{B}$ & 6,79 & 4,09 & 60,27 \\
$\mathrm{Fe} / \mathrm{P}$ & 93,2 & 64,09 & 68,77 & $\mathrm{Fe} / \mathrm{B}$ & 1,47 & 1,62 & 109,68 \\
$\mathrm{Cu} / \mathrm{P}$ & 50,66 & 86,59 & 170,93 & $\mathrm{Cu} / \mathrm{B}$ & 0,56 & 0,79 & 141,54 \\
$\mathrm{Ca} / \mathrm{K}$ & 2,65 & 0,83 & 31,12 & $\mathrm{Fe} / \mathrm{Zn}$ & 9,92 & 15,22 & 153,4 \\
$\mathrm{Mg} / \mathrm{K}$ & 0,33 & 0,10 & 31,75 & $\mathrm{Cu} / \mathrm{Zn}$ & 4,19 & 7,70 & 183,9 \\
$\mathrm{~K} / \mathrm{B}$ & 0,10 & 0,09 & 96,79 & $\mathrm{Mn} / \mathrm{Zn}$ & 60,43 & 102,63 & 169,84 \\
$\mathrm{~K} / \mathrm{Zn}$ & 0,68 & 0,69 & 102,39 & $\mathrm{Mn} / \mathrm{Fe}$ & 8,34 & 8,50 & 101,91 \\
$\mathrm{~K} / \mathrm{Mn}$ & 0,02 & 0,01 & 65,37 & $\mathrm{Cu} / \mathrm{Mn}$ & 0,12 & 0,22 & 185,65 \\
$\mathrm{Fe} / \mathrm{K}$ & 15,03 & 9,71 & 64,58 & $\mathrm{Cu} / \mathrm{Fe}$ & 0,73 & 1,23 & 169,74 \\
\hline
\end{tabular}

Quadro 4.- Normas DRIS (média, desvio padrão e coeficiente de variação) para as relações entre nutrientes dois a dois para a cultura da mangueira Tommy Atkins no Submédio São Francisco, Nordeste do Brasil
A interação entre $\mathrm{P}$ e $\mathrm{Zn}$ em solos tem sido descrita como um dos fatores que afetam a retenção e a disponibilidade de Zn em solos. De acordo com Srivastava \& Gupta (1996), um alto suprimento de $\mathrm{P}$ interfere na absorção, translocação e utilização de $Z n$, podendo induzir deficiência deste nas plantas. Supõe-se que a deficiência de $\mathrm{Zn}$ em plantas, induzida pelo $P$, ocorre: 1) pelo efeito de diluição devido à resposta ao $\mathrm{P}$; 2) pela translocação para os ramos; 3) pela interferência na função metabólica específica do $\mathrm{Zn}$ a certos sítios dentro da célula da planta; 4) pela inativação do Zn no tecido aumentar o requerimento fisiológico desse nutriente. Deficiência de $\mathrm{Zn}$ induzida por $\mathrm{P}$ aparece mesmo quando a concentração de $\mathrm{Zn}$ nas folhas é adequada. Tal deficiência aumenta com a idade da planta.

Entre as relações obtidas, o único par de elementos para o qual não se encontrou uma relação significativa foi $\mathrm{P}$ e $\mathrm{Mg}$, pelo que, é o único par para o qual não se dá norma de diagnose.

Das noventa relações consideradas entre as concentrações de nutrientes nas folhas, sessenta e duas foram significativas pelo teste F. Entre as relações da subamostra de plantas de alta produtividade cuja significância da relação entre variâncias foi estatisticamente superior, foram selecionadas quarenta e quatro relações: $P / N, N / K, N / C a$, $\mathrm{N} / \mathrm{Mg}, \mathrm{N} / \mathrm{B}, \mathrm{N} / \mathrm{Zn}, \mathrm{N} / \mathrm{Mn}, \mathrm{Fe} / \mathrm{N}, \mathrm{Cu} / \mathrm{N}, \mathrm{P} / \mathrm{K}, \mathrm{Ca} / \mathrm{P}, \mathrm{B} / \mathrm{P}, \mathrm{P} / \mathrm{Zn}$, $\mathrm{P} / \mathrm{Mn}, \mathrm{Fe} / \mathrm{P}, \mathrm{Cu} / \mathrm{P}, \mathrm{Ca} / \mathrm{K}, \mathrm{Mg} / \mathrm{K}, \mathrm{K} / \mathrm{B}, \mathrm{K} / \mathrm{Zn}, \mathrm{K} / \mathrm{Mn}, \mathrm{Fe} / \mathrm{K}, \mathrm{Cu} / \mathrm{K}$, $\mathrm{Ca} / \mathrm{Mg}, \mathrm{Ca} / \mathrm{B}, \mathrm{Ca} / \mathrm{Zn}, \mathrm{Ca} / \mathrm{Mn}, \mathrm{Ca} / \mathrm{Fe}, \mathrm{Cu} / \mathrm{Ca}, \mathrm{Mg} / \mathrm{B}, \mathrm{Mg} / \mathrm{Zn}$, $\mathrm{Mn} / \mathrm{Mg}, \mathrm{Fe} / \mathrm{Mg}, \mathrm{Cu} / \mathrm{Mg}, \mathrm{B} / \mathrm{Zn}, \mathrm{Mn} / \mathrm{B}, \mathrm{Fe} / \mathrm{B}, \mathrm{Cu} / \mathrm{B}, \mathrm{Fe} / \mathrm{Zn}$, $\mathrm{Cu} / \mathrm{Zn}, \mathrm{Mn} / \mathrm{Zn}, \mathrm{Mn} / \mathrm{Fe}, \mathrm{Cu} / \mathrm{Mn}$ e $\mathrm{Cu} / \mathrm{Fe}$ (Quadro 3), cujos valores das normas estão no Quadro 4. Raghupathi et al. (2004), para a manga, cv. Totapuri selecionaram quarenta e cinco expressões de nutrientes como normas de diagnose.

\section{Conclusão}

Foram geradas as normas DRIS para a mangueira Tommy Atkins, aplicáveis à diagnose do estado nutricional dessa cultura nas condições de clima e solo do Submédio São Francisco no Nordeste do Brasil, estabelecidas com base na subamostra de plantas de alta produtividade, envolvendo os seguintes pares de nutrientes: $\mathrm{P} / \mathrm{N}, \mathrm{N} / \mathrm{K}, \mathrm{N} / \mathrm{Ca}, \mathrm{N} / \mathrm{Mg}$, $\mathrm{N} / \mathrm{B}, \mathrm{N} / \mathrm{Zn}, \mathrm{N} / \mathrm{Mn}, \mathrm{Fe} / \mathrm{N}, \mathrm{Cu} / \mathrm{N}, \mathrm{P} / \mathrm{K}, \mathrm{Ca} / \mathrm{P}, \mathrm{B} / \mathrm{P}, \mathrm{P} / \mathrm{Zn}, \mathrm{P} / \mathrm{Mn}$, $\mathrm{Fe} / \mathrm{P}, \mathrm{Cu} / \mathrm{P}, \mathrm{Ca} / \mathrm{K}, \mathrm{Mg} / \mathrm{K}, \mathrm{K} / \mathrm{B}, \mathrm{K} / \mathrm{Zn}, \mathrm{K} / \mathrm{Mn}, \mathrm{Fe} / \mathrm{K}, \mathrm{Cu} / \mathrm{K}$, $\mathrm{Ca} / \mathrm{Mg}, \mathrm{Ca} / \mathrm{B}, \mathrm{Ca} / \mathrm{Zn}, \mathrm{Ca} / \mathrm{Mn}, \mathrm{Ca} / \mathrm{Fe}, \mathrm{Cu} / \mathrm{Ca}, \mathrm{Mg} / \mathrm{B}, \mathrm{Mg} / \mathrm{Zn}$, $\mathrm{Mn} / \mathrm{Mg}, \mathrm{Mg} / \mathrm{Fe}, \mathrm{Cu} / \mathrm{Mg}, \mathrm{B} / \mathrm{Zn}, \mathrm{Mn} / \mathrm{B}, \mathrm{Fe} / \mathrm{B}, \mathrm{Cu} / \mathrm{B}, \mathrm{Fe} / \mathrm{Zn}$, $\mathrm{Cu} / \mathrm{Zn}, \mathrm{Mn} / \mathrm{Zn}, \mathrm{Mn} / \mathrm{Fe}, \mathrm{Cu} / \mathrm{Mn}$ e $\mathrm{Cu} / \mathrm{Fe}$.

Agradecimentos À Coordenadoria de Aperfeiçoamento de Pessoal (CAPES) pela bolsa de estudos fornecida; ao Centro de Apoio ao Desenvolvimento Científico e Tecnológico (CADCT) da SEPLANTEC-BA (atualmente FAPESB) e ao Banco do Nordeste, BNB, pelo apoio financeiro; à EMBRAPA/CPATSA, ao Instituto de Pesquisas Agropecuárias de Pernambuco, IPA, à VALEXPORT/GMV, CODEVASF, $3^{\mathrm{a}}$. e $6^{\mathrm{a}}$. SR, ATER/PSNC, FRUITFORT, FAMA, CAJ, NOVA FRONTEIRA, AGROVALE, EBRAZ, FRUTIVALE, LASTRO AGRÍCOLA, UPA AGRÍCOLA, produtores de manga, engenheiros agrônomos, técnicos agrícolas e todos que contribuíram na execução do trabalho.

\section{Bibliografia}

Alvarez V., V. H. \& Leite, R. de A. (1999). Fundamentos estatísticos das fórmulas usadas para o cálculo dos índices DRIS. Boletim Informativo da Sociedade Brasileira de Ciência do Solo 24. 1: 13-15. 
Bailey, J. S.; Beattie, J. A. M. \& Kilpatrick, D. J. (1997). The diagnosis and recommendation integrated system (DRIS) for diagnosing the nutrient status of grassland swards I. Model establishment. Plant and Soil. 197: 127-135.

Baldock, J. O. \& Schulte, E. E. (1996). Plant analysis with standardized scores combines DRIS and sufficiency range approaches for corn. Agronomy Journal. 88: 448-456.

Camargo, P. N. de \& Silva, O. (1990). Manual de adubação foliar. S. Paulo, Herba.

Dechen, A. R.; Haag, H. P. \& Carmello, Q. A. de C. (1991). Mecanismos de absorção e de translocação de micronutrientes. In: Ferreira, M. E. \& Cruz, M. C. P. Simpósio sobre micronutrientes na agricultura. Jaboticabal. 1991. Anais. Piracicaba: Potafos/CNPq :79-97.

Embrapa - Cpatsa .Dados meteorológicos. Disponível em: <http://www.cpatsa.embrapa.br/index.php?op=eabeb>. [Acesso em 30 setembro 2008]

Jacomine, P.K.T., Cavalcanti, A.C., Ribeiro, M.R., Montenegro, J. O., Burgos, N., Filho, H.F.R. de M. \& Formiga, R.A. (1976). Levantamento exploratório reconhecimento da margem esquerda do rio São Francisco, Estado da Bahia. Recife, EMBRAPA, Serviço Nacional de Levantamento e Conservação de Solos. (Brasil. EMBRAPA, SNLCS. Boletim Técnico, 38). Brasil, SUDENE. DRN. Divisão de Recursos Renováveis, 7.

Hallmark, W.B. \& Beverly, R.B. (1991). Review - an update in the use of the Diagnosis and Recommendation Integrated System. Journal of Fertilizers Issues. 8: 74-88.

Hundal, H.S., Singh, D. \& Brar, J.S. (2005). Diagnosis and recommendation integrated system for monitoring nutrient status of mango trees in submountainous area of Punjab, India. Communications In Soil Science and Plant Analysis. 36, 15-16: 2085-2099.

Jones, C.A. (1981). Proposed modifications of the diagnosis and recommendation integrated system (DRIS) for interpreting plant analysis. Commun. Soil Sci. and Plant Anal 12: 785-974

Jones Jr, J.B. (1993). Modern interpretation systems for soil and plant analysis in the USA. Australian J. of Exp. Agric. 33: $1039-1043$.

Malavolta, E., Vitti, G.C. \& Oliveira, S.A. (1997). Avaliação do estado nutricional das plantas - princípios e aplicações. 2.ed. rev. e atual. Piracicaba, POTAFOS.

Meldal - Johnsen, A. \& Sumner, M.E. (1980). Foliar diagnostic norms for potatoes. Journal of Plant Nutrition. 2: 569-576.

Payne, G.G.; Rechcigl, J.E. \& Stephenson, R.L. (1990). Development of Diagnosis and Recommendation Integrated System norms for Bahiagrass. Agronomy Journal. 82: 930934.

Peverill, K.I. (1993). Soil testing and plant analysis in Australia. Australian Journal of Experimental Agriculture. 33: 963-71.
Quaggio, J.A. (1996). Adubação e calagem para a mangueira e qualidade dos frutos. In: São José, A. R.; Souza, I. V. B.; Filho, J. M. \& Morais, O. M. Manga, tecnologia de produção e mercado. Vitória da Conquista. BA, DFZ/UESB 106-135.

Raghupathi, H.B. \& Bhargava, B.S. (1997). Preliminary diagnostic soil fertility norms for Alphonso mango (Mangifera indica L.). Journal of the Indian Society of Soil Science. 45, 3: $534-536$

Raghupathi, H.B. \& Bhargava, B.S. (1999). Preliminary nutrient norms for 'Alphonso' mango using diagnosis and recommendation integrated system. Indian Journal of Agricultural Sciences. 69, 9: 648-650.

Raghupathi, H.B, Reddy, Y.T.N, Rein, M.K. \& Bhargava, B.S. (2004). Diagnosis of nutrient imbalance in mango by DRIS and PCA approaches. Journal of Plant Nutrition. 27, 7: 11311148.

Raj, G.B. \& Rao, A.P. (2006). Identification of yield-limiting nutrients in mango through DRIS indices. Communications In Soil Science And Plant Analysis. 37, 11-12: 1761-1774.

Reis Jr., R. dos A. (1999). Diagnose nutricional da cana - de - açúcar com uso do sistema integrado de diagnose e recomendação (DRIS). Campo dos Goytacazes, RJ (Tese D.S.)

Samra, J.S. \& Arora, Y.K. (1997). Mineral nutrition. En: Litz, R. E. The mango: botany, production and uses. $C A B$ International, New York.

Schaffer, B., Larson, K.D., Snyder, G.H. \& Sanchez, C.A. (1988). Identification of mineral deficiencies associated with mango decline by DRIS. Hortscience. 23, 3: $617-619$.

Srivastava, P.C. \& Gupta, U.C. (1996). Trace elements crop production. Science Publishers, Inc., Lebanon.

Sumner, M.E. (1999). The use and misuse of the Diagnosis and Recommendations Integrated System (DRIS) in foliar diagnosis. Anais do Simpósio sobre Monitoramento nutricional para a recomendação de adubação de culturas São Paulo, Piracicaba, Potafos, 14 - 16 de abril. (CD Rom).

Wadt, P.G.S. (1995). Software para cálculo de índices DRIS, índices IBN mediante diferentes fórmulas. UFV, Viçosa, MG. (Dois disquetes 3,5")

Wadt, P.G.S., Silva, D.J., Maia, C.E., Tomé Júnior, J.B.,Pinto, P.A. da C. \& Machado, P.L. de O. de A. (2007). Modelagem de funções no cálculo dos índices DRIS, Pesq. Agropec. Bras. 42, 1: 57-64.

Walworth, J.L. \& Sumner, M.E. (1987).The diagnosis and recommendation integrated system (DRIS). Advances in Soil Science. 6:149-188. 